\title{
Novel roles for GAPDH in cell death and carcinogenesis
}

\author{
A Colell ${ }^{*, 1}$, DR Green ${ }^{2}$ and J-E Ricci ${ }^{*, 3,4,5}$
}

Growing evidence points to the fact that glucose metabolism has a central role in carcinogenesis. Among the enzymes controlling this energy production pathway, glyceraldehyde-3-phosphate dehydrogenase (GAPDH) is of particular interest. Initially identified as a glycolytic enzyme and considered as a housekeeping gene, this enzyme is actually tightly regulated and is involved in numerous cellular functions. Particularly intriguing are recent reports describing GAPDH as a regulator of cell death. However, its role in cell death is unclear; whereas some studies point toward a proapoptotic function, others describe a protective role and suggest its participation in tumor progression. In this study, we highlight recent findings and discuss potential mechanisms through which cells regulate GAPDH to fulfill its diverse functions to influence cell fate.

Cell Death and Differentiation (2009) 16, 1573-1581; doi:10.1038/cdd.2009.137; published online 25 September 2009

Initially identified as a 'simple' glycolytic enzyme, glyceraldehyde 3-phosphate dehydrogenase (GAPDH) is tightly regulated at both transcriptional and posttranslational levels and has numerous cellular functions ascribed to it. ${ }^{1,2}$ Among these, the role of GAPDH in cell death remains poorly understood. Initially identified as a proapoptotic agent, it is perhaps paradoxical that a majority of human tumors overexpress it. Recent findings underline its role in tumorigenesis, as well as in tumor progression and cell survival. The aim of this review is to provide an overview of how GAPDH functions, how it can be modulated and its different roles in controlling cell life and death.

\section{GAPDH is a Multifunctional Protein}

GAPDH is one of the enzymes involved in the ubiquitous process of glycolysis. GAPDH specifically catalyzes the simultaneous phosphorylation and oxidation of glyceraldehyde-3-phosphate to 1,3-biphosphoglycerate, using NAD ${ }^{+}$ as the electron acceptor. GAPDH comprises a polypeptide chain of 335 amino acids. Structural studies identified two regions, namely the glyceraldehyde-3-phosphate catalytic site and the $\mathrm{NAD}^{+}$binding site, a primary structure known as the Rossmann fold, which is also required for the activity of other dehydrogenases. ${ }^{1}$ The glycolytic function mainly relies on critical amino acids that include $\mathrm{Cys}^{152}$ and $\mathrm{His}^{179}$, and on its tetrameric structure composed of four identical $37-\mathrm{kDa}$ subunits. ${ }^{1}$

Besides its conventional metabolic role, a number of studies have identified the participation of GAPDH in diverse cellular functions. Many of these roles are dependent on the ability of GAPDH to bind different macromolecules in the cell. Early reports first identified the fusogenic properties of GAPDH, ${ }^{3}$ whereas subsequent observations showed its physiological significance in endocytosis and nuclear membrane assembly. ${ }^{4,5}$ Membrane transport between the endoplasmic reticulum and the Golgi complex has also been described to require the binding of GAPDH to the small GTPase Rab2 present in pre-Golgi intermediates. ${ }^{6}$ In addition, an association of GAPDH with microtubules seems to have an essential role in tubulin bundling and cytoskeletal dynamics. ${ }^{7,8}$ These studies show that ATP induces the dissociation of the GAPDH tetramer and inhibits its bundling activity. ${ }^{8}$ Recently, in vitro microtubule binding assays showed that Rab2 associates with microtubules but only when GAPDH and atypical $\mathrm{PKC} \tau$ are present. ${ }^{9}$ This association stimulates the recruitment of the motor protein dynein, which in turn regulates microtubule motility and cargo transport. $^{9}$

GAPDH also interacts with nucleic acids. Early studies noted the high affinity of the enzyme for transfer RNA (tRNA),

\footnotetext{
${ }^{1}$ Department of Cell Death and Proliferation, Institut d'Investigacions Biomèdiques de Barcelona, Consejo Superior de Investigaciones Científicas and Liver Unit, Hospital Clinic i Provincial, Centro de Investigaciones Biomédicas Esther Koplowitz, and CIBEREHD, IDIBAPS, Barcelona, Spain; ${ }^{2}$ Department of Immunology, St. Jude Children's Research Institute, Memphis, TN 92105, USA; ${ }^{3}$ Inserm, U895, Centre Méditerranéen de Médecine Moléculaire (C3M), équipe 3 AVENIR, Nice, F-06204 Cedex 3, France; ${ }^{4}$ Université de Nice-Sophia-Antipolis, Faculté de Médecine, Nice, F-06107 Cedex 2, France and ${ }^{5}$ Centre Hospitalier Universitaire de Nice, département d'Anesthésie Réanimation, Nice, F-06202 Cedex 3, France

*Corresponding authors: A Colell, Department of Cell Death and Proliferation, Institut d'Investigacions Biomèdiques de Barcelona (IIBB-CSIC), Rosselló 161, 08036Barcelona, Spain. Tel: + 003493363 8310; Fax: + 003493363 8301; E-mail: anna.colell@iibb.csic.es or

J-E Ricci, équipe-3 Avenir, Inserm U895, Faculté de Médecine de Nice, 28 Av de Valombrose, 06107 Nice cedex 02, France.

Tel: + 00334933770 17; Fax + 00334938178 52; E-mail: ricci@unice.fr

Keywords: cell death; glycolysis; GAPDH; mitochondria; cancer

Abbreviations: GAPDH, glyceraldehyde-3-phosphate dehydrogenase; CSF-1, colony-stimulating factor-1; H2B, S-phase-dependent histone-2B

Received 06.5.09; revised 31.7.09; accepted 04.8.09; Edited by G Melino; published online 25.9.09
} 
and its ability to discriminate between wild-type and tRNA mutants that are defective in nuclear export, suggesting that GAPDH may participate in tRNA export. ${ }^{10}$ Subsequent studies have further characterized GAPDH as an RNAbinding protein, with preference to AU-rich elements, and localized the binding activity to the Rossmann fold of the enzyme. ${ }^{11}$ Recently, these findings have been confirmed and extended, demonstrating that, through this interaction, GAPDH regulates mRNA stability and consequently controls the expression of proteins, such as endothelin- $1^{12}$ and colonystimulating factor-1 (CSF-1). ${ }^{13}$

In addition to its cytosolic and membrane roles, GAPDH shows several nuclear functions. It is part of the OCA-S complex, a multicomponent Oct-1 coactivator that is essential for S-phase-dependent histone-2B (H2B) transcription. ${ }^{14}$ GAPDH binds directly to Oct-1, is selectively recruited to the H2B promoter in the S-phase and seems to have an intrinsic activation domain that interacts with an as yet unidentified component of the basal RNA polymerase II transcription machinery. ${ }^{14}$ Studies in fission yeast showed that GAPDH interacts with the Rbp7 subunit of RNA polymerase II, further supporting its participation in transcription. ${ }^{15}$ Its role in DNA repair was suggested by the observation that GAPDH monomers exhibit a uracyl DNA glycosylase activity that is cell-cycle regulated. ${ }^{16}$ Further studies designed to elucidate cellular response to mercaptopurines showed the formation of a DNA-protein complex containing GAPDH that binds thioguanylated DNA and may act as a sensor of structural DNA alterations. ${ }^{17}$ Moreover, it has recently been described that GAPDH may have a protective effect on telomere length after ceramide exposure. ${ }^{18}$

\section{Mechanisms of GAPDH Regulation}

The exact mechanisms by which GAPDH performs its non-glycolytic functions remain largely obscure. The GAPDH gene is localized on chromosome 12 in humans and on chromosome 6 in mice, and encodes a single mRNA species leading to the production of a single protein. Therefore, the functional diversity of GAPDH is probably not the result of differential RNA processing but is more likely a consequence of posttranslational events.

Although GAPDH is widely used as an internal control, its mRNA and protein levels vary in response to various stimuli. Indeed, the GAPDH gene and protein are actively regulated on cell proliferation. ${ }^{19-21}$ GAPDH is the target of different transcription factors, and various control regions have been identified in its promoter, including hypoxia and insulinresponsive elements ${ }^{22,23}$ (Figure 1). In the context of cancer, hypoxia is of particular interest. Tumor cells maintain a high proliferation rate and consumption of nutrients and oxygen that often overcomes the support capacity of existing local blood vessels. ${ }^{24}$ As a result, areas with low oxygen develop in most solid tumors, ${ }^{25,26}$ and cells have diverse mechanisms to adjust to hypoxia. Although adaptation to an acute decrease in oxygen levels is mainly mediated by reversible posttranscriptional modifications such as phosphorylation, under chronic deprivation, cells induce the expression of new genes that allow the tumor to adapt to these stressful conditions. Hypoxic gene transcription is mediated by various transcription factors, including $\mathrm{AP}-1, \mathrm{NF}-\kappa \mathrm{B}, \mathrm{CREB}$ and $\mathrm{p} 53$, among others. Particularly relevant is the transcription factor $\mathrm{HIF}-1,{ }^{27}$ of which more than 100 direct targets have been identified. Among these, the expression of glycolytic enzymes, ${ }^{28,29}$ including $\mathrm{GAPDH}$, is upregulated on oxygen deprivation. ${ }^{30,31}$

Protein levels can also be affected by changes in degradation. GAPDH is among the $30 \%$ of soluble cytosolic cellular proteins that contain a KFERQ amino-acid sequence that targets those proteins for lysosomal degradation ${ }^{32}$ (Figure 1). This process of chaperone-mediated autophagy is part of the cellular quality control system that is essential for cellular response to stress. ${ }^{33,34}$

However, the wide functional diversity of GAPDH cannot be explained only by changes in protein levels; indeed, other posttranslational events may have a key role in regulating GAPDH glycolitic and non-glycolitic functions (Figure 1). In one study, phosphorylation by the muscle-specific isoform of $\mathrm{Ca}^{2+} /$ calmodulin-dependent protein kinase II increased GAPDH glycolitic activity by 3.4 -fold and allowed the assembly of glycogen-mobilizing and glycolytic enzymes at the sarcoplasmic reticulum (SR) membrane in response to calcium signaling. ${ }^{35}$ It was suggested that this could serve to modulate ATP and NADH levels at the SR, thereby allowing the regulation of calcium transport processes. GAPDH phosphorylation by $\mathrm{PKC} \tau / \lambda$ has also been suggested to target the enzyme to pre-Golgi intermediates, enhancing its participation in microtubule dynamics. ${ }^{6,36,37}$

GAPDH is a key redox-sensitive protein, the activity of which is largely affected by covalent modifications by oxidants at its highly reactive Cys ${ }^{152}$ residue. The protein is inhibited when it undergoes $S$-nitrosylation by nitric oxide (NO), ${ }^{38,39} \mathrm{NAD}^{+}$covalent linkage on $S$-nitrosylation, ${ }^{39}$ nitroalkylation by nitrated fatty acids, ${ }^{40} S$-glutathionylation by gluthatione and by $\mathrm{NO}^{41}$ as well as extensive oxidation by $\mathrm{H}_{2} \mathrm{O}_{2}$ or peroxynitrite. ${ }^{42,43}$ These oxidative changes not only affect the glycolytic function but also stimulate the participation of GAPDH in cell death (described in the next section).

Another level of regulation is the translocation of the enzyme to the nucleus, which can be cell-cycle dependent $^{14,18}$ or triggered in response to cellular stress. ${ }^{44-49}$ Similarly, serum withdrawal was described to induce an accumulation of GAPDH in the nucleus. ${ }^{50}$ However, in contrast to other cell death stimuli, this nuclear translocation is a reversible process that could be recovered on serum addition, likely triggered by survival signals. Recently, a novel exportin1 or chromosome region maintenance (CRM)1dependent nuclear export signal was identified in the C-terminal GAPDH domain. ${ }^{51}$ Truncation or mutation of this sequence abrogated CRM1 binding and caused nuclear accumulation of GAPDH. Nuclear targeting can also be modulated by posttranslational changes. $O$-linked $N$-acetylglucosamine modifications of GAPDH (O-GlcNAcylation mainly on $\mathrm{Thr}^{227}$ ) were reported as being able to disrupt the tetrameric form, enabling its nuclear translocation ${ }^{52}$ (Figure 1). This finding contrasts with that of Hara et al., ${ }^{38}$ which described that $S$-nitrosylation of GAPDH allowed binding with Siah1, leading to the nuclear translocation of GAPDH dependent on Siah1 NLS. However, as the interaction of the $O$-GIcNAcylation form of GAPDH with Siah1 was not tested, it 


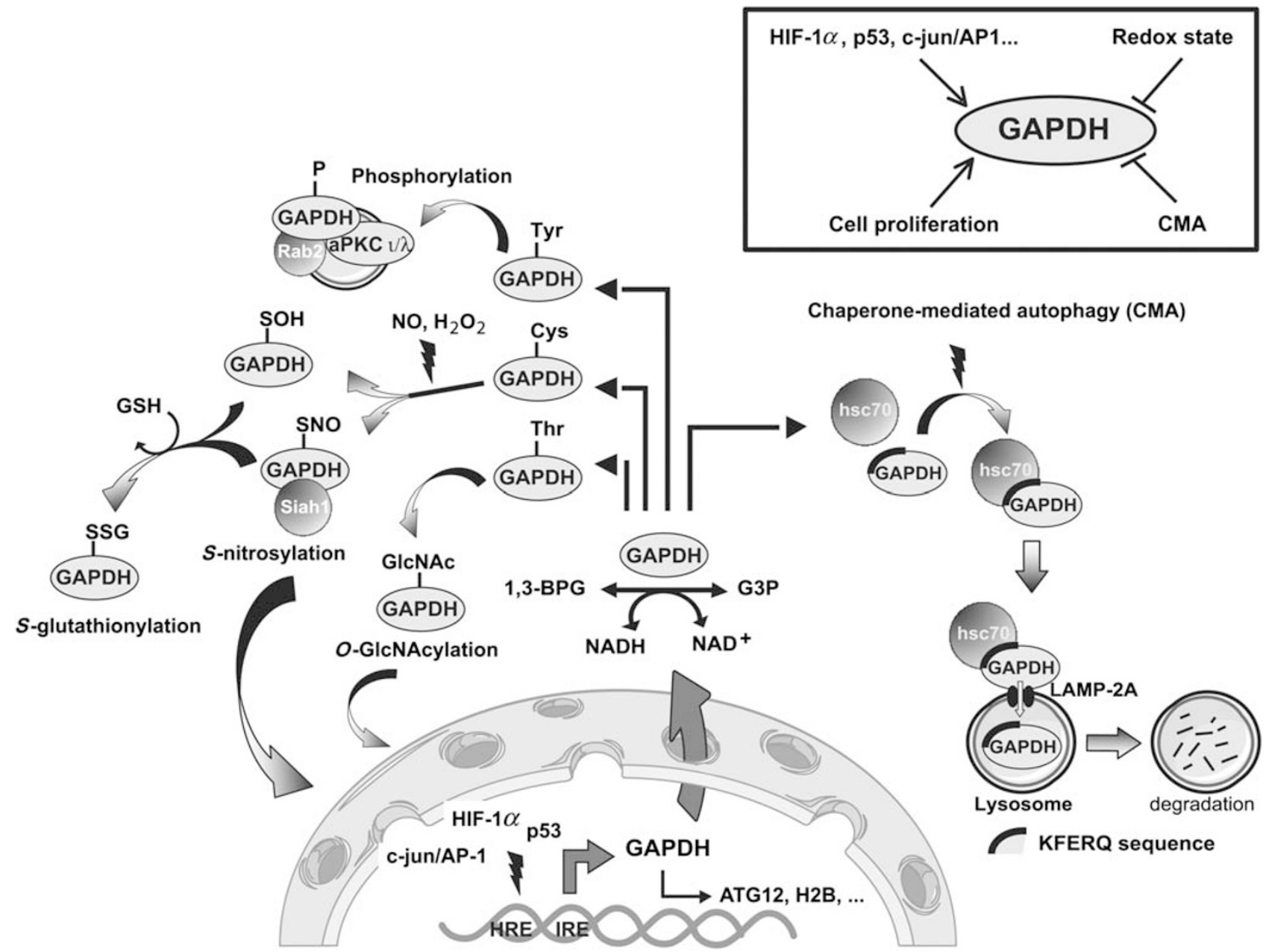

Figure 1 Regulatory mechanisms of GAPDH. Cellular GAPDH content is modulated by several biological processes (see inset). Its expression is highly dependent on the proliferative state of the cell and can also be regulated by transcription factors, such as hypoxia-inducible factor 1 (HIF-1 $\alpha$ ), p53 or c-jun/AP1. Indeed, different control regions have been identified on its promoter (HRE, hypoxia response element; IRE, insulin response element) that may regulate its levels in certain circumstances, such as low oxygen supply. In the cytosol, levels of the glycolytic enzyme, which catalyzes the conversion of glyceraldehyde-3-phosphate (G3P) into 1,3-biphosphoglycerate (1,3-BPG), can be affected by changes in its lysosomal degradation. Under conditions of cellular stress, chaperone-mediated autophagy is activated; GAPDH contains a KFERQ motif that is selectively recognized by the chaperone heat shock cognate protein of $70 \mathrm{kDa}$ (hsc70). This interaction targets the complex to the lysosomal membrane, where it binds to the lysosome-associated membrane protein type 2A (LAMP-2A) that acts as a receptor for this pathway. At the posttranslational level, GAPDH can undergo different modifications, which may determine some of its non-glycolytic functions. Tyrosine (Tyr) phosphorylation by the atypical protein kinase $\mathrm{C} \tau / \lambda$ (aPKC $\tau / \lambda$ ) is required for its association with Rab2 and for the transport between the endoplasmic reticulum and the Golgi complex. Hydrogen peroxide $\left(\mathrm{H}_{2} \mathrm{O}_{2}\right)$ and nitric oxide $(\mathrm{NO})$ both act on the active site cysteine 152 (Cys). The irreversible oxidation of the Cys residue can be prevented by S-glutathionylation, in which the sulfydryl group (-SH) conjugates with glutathione (GSH). S-nitrosylation facilitates its binding to Siah1 and also the translocation of the complex to the nucleus. In addition, GAPDH can undergo O-linked $\beta$-N-acetylglucosamine glycosylation (O-GlcNAcylation) at threonine residues, which also may mediate its nuclear migration by disrupting the tetrameric conformation of the enzyme. Once in the nucleus, GAPDH can participate in DNA repair mechanisms and in the transcriptional regulation of proteins, including ATG12 autophagy-related 12 homolog and histone $\mathrm{H} 2 \mathrm{~B}$

is conceivable that a common mechanism of Siah1-dependent nuclear import of GAPDH may exist.

Overall, these data strongly support the notion that posttranslational changes of GAPDH can affect its cellular functions, opening an important avenue that requires further work.

\section{GAPDH as a Proapoptotic Agent}

Evidence for the potential apoptotic role of GAPDH first came from studies on cultured cerebellar neurons, ${ }^{46-48,53}$ in which an increase in GAPDH levels and its subsequent translocation to the nucleus preceded neuronal death induced by culture aging or cytosine arabinonucleoside exposure. Antisense oligonucleotides directed against GAPDH mRNA exerted a protective effect, and either antisense treatment or incubation with protein synthesis inhibitors prevented its appearance in the nucleus. ${ }^{47,53}$ This suggested that nuclear GAPDH is mainly derived from new protein synthesis and may function in cell death. These initial observations have been extended to non-neuronal cells that are exposed to several apoptotic stimuli (summarized in Table 1).

Nuclear accumulation of GAPDH precedes apoptotic features that are antagonized by the overexpression of $\mathrm{Bcl}-2 .{ }^{44,54}$ Once in the nucleus, GAPDH loses its catalytic 
Table 1 Participation of GAPDH in cell death and carcinogenesis

\begin{tabular}{|c|c|c|c|}
\hline Cell lines & Stimulus & Proposed mechanisms & References \\
\hline \multicolumn{4}{|l|}{ Participation in apoptosis } \\
\hline $\begin{array}{l}\text { HEK293; Raw264.7; } \\
\text { cerebellar neurons }\end{array}$ & Staurosporin; LPS/IFN $\gamma ;$ NMDA & $\begin{array}{l}\text { S-nitrosylation of GAPDH and } \\
\text { nuclear translocation }\end{array}$ & Hara et al. ${ }^{38}$ \\
\hline Cerebrocortical neurons & Culture aging & Increased expression of GAPDH & Ishitani et al. ${ }^{45}$ \\
\hline Cerebellar neurons & Culture aging; AraC; low $\mathrm{K}^{+}$ & $\begin{array}{l}\text { Increased expression of GAPDH } \\
\text { and nuclear translocation }\end{array}$ & $\begin{array}{l}\text { Ishitani et al. }{ }^{46} \text {; Ishitani et al. } .^{47} \text {; } \\
\text { Saunders et al. }{ }^{48} \text {; Ishitani and } \\
\text { Chuang }{ }^{53} \text {; Saunders et al. } .^{55} \text {; }\end{array}$ \\
\hline \multirow{2}{*}{$\begin{array}{l}\text { S49; primary thymocytes; PC12; } \\
\text { cerebrocortical neurons } \\
\text { Neuroblastoma cells (mNB41A3); } \\
\text { R6 fibroblasts }\end{array}$} & $\begin{array}{l}\text { Dexamethasone; NGF; culture } \\
\text { aging }\end{array}$ & Nuclear translocation & Sawa et al. ${ }^{49}$ \\
\hline & $\begin{array}{l}\text { Staurosporin; MG132; } \mathrm{H}_{2} \mathrm{O}_{2} \text {; } \\
\text { FeCN }\end{array}$ & Nuclear translocation & Dastoor and Dreyer ${ }^{44}$ \\
\hline Mesencephalic neurons & MPP+ & Nuclear translocation & Fukuhara et al. ${ }^{103}$ \\
\hline Neuroblastoma cells (SH-SY5Y) & Dopaminergic neurotoxin & Nuclear translocation & Maruyama et al. ${ }^{104}$ \\
\hline Hepatocytes & TGF- $\beta$ & Nuclear translocation & Barbini et al. ${ }^{105}$ \\
\hline HeLa & NO donor (NOC18) & Nuclear GAPDH aggregates & Nakajima et al. ${ }^{56}$ \\
\hline $\begin{array}{l}\text { Raw264.7; macrophages; } \\
\text { HEK293; neuroblastoma cells }\end{array}$ & LPS/IFN $\gamma$; NO donor (GSNO) & $\begin{array}{l}\text { Induction of p53 by GAPDH- } \\
\text { mediated activation of P300/CBP }\end{array}$ & Sen et al. ${ }^{63}$ \\
\hline $\begin{array}{l}\text { (SH-SY5Y) } \\
\text { Follicular thyroid cell; KTC2 }\end{array}$ & TRAIL & $\begin{array}{l}\text { S-nitrosylation of GAPDH and } \\
\text { nuclear translocation }\end{array}$ & Du et al. ${ }^{66}$ \\
\hline HeLa; HEK293 & $\begin{array}{l}\text { Staurosporin; etoposide; } \\
\text { Lonidamine }\end{array}$ & $\begin{array}{l}\text { GAPDH-induced mitochondrial } \\
\text { permeabilization }\end{array}$ & Tarze et al. ${ }^{67}$ \\
\hline Neuroblastoma cells (N2a) & Mutant huntingtin (mHtt) & $\begin{array}{l}\text { GAPDH-mediated translocation } \\
\text { of } \mathrm{mHtt}\end{array}$ & Bae et al. ${ }^{74}$ \\
\hline HT22 & $\mathrm{A} \beta$ peptides & $\begin{array}{l}\text { Disulfide-linkage and nuclear } \\
\text { accumulation of GAPDH }\end{array}$ & Cumming and Schubert ${ }^{76}$ \\
\hline \multicolumn{4}{|c|}{ Participation in carcinogenesis or protection from cell death } \\
\hline HeLa, primary MEFs & $\begin{array}{l}\text { Etoposide, staurosporine, } \\
\text { actinomycine } D \text {, oncogenes }\end{array}$ & $\begin{array}{l}\text { Protection from CICD by glycolysis } \\
\text { increase and autophagy induction }\end{array}$ & Colell et al. ${ }^{87}$ \\
\hline K562, JURL-MK1 & Imatinib mesylate & Inhibition of CICD & Lavallard et al. $^{81}$ \\
\hline Jurkat, Molt4, primary ALL cells & Prednisolone & $\begin{array}{l}\text { GAPDH downregulation sensitizes } \\
\text { resistant cells to treatment }\end{array}$ & Hulleman et al. ${ }^{95}$ \\
\hline NOSE.1, Hey & & GAPDH stabilizes CSF-1 mRNA & Zhou et al. ${ }^{13}$ \\
\hline $\begin{array}{l}\text { HCT116, DLD1, primary } \\
\text { lung fibroblastes (LF1) }\end{array}$ & $\begin{array}{l}\text { Methyl methane sulfonate, } \\
\text { bleomycin }\end{array}$ & GAPDH interaction with APE1 & Azam et al. ${ }^{97}$ \\
\hline
\end{tabular}

activity and becomes resistant to standard extraction procedures. ${ }^{44,49}$ Further studies have shown that induction of cell death is accompanied by the appearance of nuclear alkaline forms of the enzyme distinct from cytosolic species. ${ }^{55}$ Although these findings would suggest the involvement of novel GAPDH forms in cell death, it is worth noting that it has also been described that the presence of basic forms of GAPDH is cell-cycle regulated and exerts a protective role against telomere shortening induced by ceramide or chemotherapeutic agents. ${ }^{18}$ Thus, it is conceivable that the participation of GAPDH in cell death may be determined by other posttranslational events. Further support for the existence of these regulatory mechanisms was the observation that apoptotic treatment markedly reduced sodium nitroprusside-induced $\mathrm{NAD}^{+}$labeling of nuclear $\mathrm{GAPDH}$, suggesting that the active site of GAPDH may be covalently modified, denatured or improperly folded. ${ }^{55}$ In this regard, recent studies have shown that sustained exposure to oxidants, through the formation of intermolecular disulfide bonds, induced insoluble amyloid-like GAPDH aggregates that promote cell death ${ }^{56}$ (Figure 2). This irreversible oxidation can be prevented by protein $S$-thiolation, in which protein sulphydryl groups form mixed disulfides with low-molecularweight thiols, such as glutathione. ${ }^{57,58}$ Moreover, studies in Caenorhabditis elegans indicated that redox regulation of
GAPDH can counteract oxidative stress by repressing the glycolytic pathway and consequently rerouting the metabolic flux to maintain an optimal NADPH/NADP ${ }^{+}$ratio, through the pentose cycle. ${ }^{59}$ Strikingly, the redox-sensitive cysteine residue of the glycolytic enzyme also has an essential role in signaling pathways that sense oxidative stress in the phospho-relay signaling of the fission yeast Schizosaccharomyces pombe ${ }^{60}$ (Figure 2). In this system, peroxide stress signals are transmitted from Mak2/3 sensor kinases to the Mpr1 histidine-containing phosphotransfer protein and finally to the Mcs4 response regulator, ultimately activating a MAP kinase cascade. In response to oxidative stress, the transient oxidization of GAPDH facilitates its association with the Mcs4 response regulator, and this represents an essential step for the interaction between Mcs4 and Mpr1. ${ }^{60}$

GAPDH has also been reported as a sensor of $\mathrm{NO}$ stress. ${ }^{61,62} \mathrm{NO}$ causes $S$-nitrosylation of GAPDH at its active site, increasing the binding to Siah1 (an E3 ubiquitin ligase) ${ }^{38,62}$ (Figure 2), the nuclear localization signal of which mediates translocation of GAPDH. In turn, GAPDH in the nucleus stabilizes Siah1, facilitating its degradation of nuclear proteins. ${ }^{38,62}$ Further studies have shown that nuclear $S$-nitrosylated GAPDH is acetylated at $\mathrm{Lys}^{160}$ by the acetyltransferase p300/CREB-binding protein (CBP), ${ }^{63}$ which enhances the ability of GAPDH to stimulate auto-acetylation 


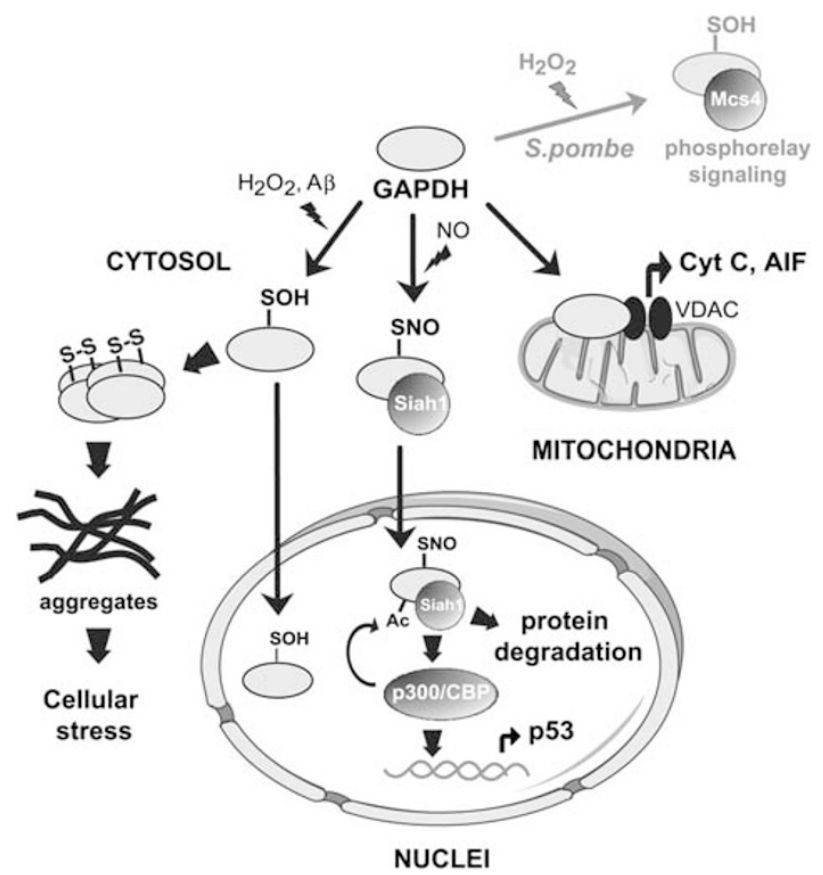

Figure 2 GAPDH participation in cell death. GAPDH can trigger oxidative stress-mediated cell death. Exposure to oxidants or amyloid- $\beta$ peptides $(A \beta)$ induces an irreversible oxidation of cysteine residues that favor intermolecular disulfide bonds and the subsequent formation of cytosolic aggregates. This insoluble protein may ultimately promote cellular stress. Oxidative modifications can also target GAPDH to the nucleus. S-nitrosylation of the enzyme increases binding to Siah1, which mediates its nuclear translocation. GAPDH stabilizes Siah1, enhancing the activity of this ubiquitin ligase and the proteasome-mediated degradation of nuclear proteins. Nuclear GAPDH is acetylated by the p300/CREBbinding protein (CBP), which in turn stimulates the catalytic activity of $\mathrm{p300/CBP}$. Consequently, downstream targets of p300/CBP, such as p53, can be activated and cause cell death. GAPDH has been also localized in the mitochondria, in which its binding to the voltage-dependent anion channel (VDAC) has been suggested to promote the release of proapoptotic proteins, such as cytochrome $c$ (CytC) and apoptosis-inducing factor (AIF). In prokaryotes, the glycolytic enzyme has been involved in signaling pathways that sense oxidative stress. The oxidation of GAPDH facilitates its association with the Mcs4 response regulator, an essential step in the phosphorelay signaling that ultimately activates a MAP kinase cascade

and catalytic activity of p300/CBP, and, consequently, downstream targets such as p53 are activated. Interestingly, both Siah-1, with a specific p53 response element within its second intron, and GAPDH have been shown to be upregulated by p53, ${ }^{64,65}$ pointing to the existence of auto-amplifying loops. The involvement of $S$-nitrosylation mechanisms in GAPDHmediated cell death is further supported by studies in thyroid cancer cell lines in which $S$-nitrosylation and nuclear translocation of GAPDH are observed after TNF-related apoptosis-inducing ligand (TRAIL) exposure. ${ }^{66}$ Knockdown of GAPDH with small-interfering RNA partially prevented the apoptotic effect of TRAIL, although NO synthase stimulation and production of NO were not attenuated. ${ }^{66}$

After cell death induction, GAPDH levels were also found to apparently increase in the mitochondria; ${ }^{47,48}$ however, its role within this organelle remains elusive. Recent studies in isolated mitochondria have suggested that GAPDH interacts with the voltage-dependent anion channel 67 (Figure 2), inducing a cyclosporin A-inhibitable permeability transition and the release of proteins, such as cytochrome $c$ and apoptosis-inducing factor. ${ }^{67}$ It is worth noting that these observations are based on the use of exogenous GAPDH at concentrations that may not reflect cellular levels. Indeed, only a small portion of total cellular GAPDH is found in the mitochondrial fraction after induction of cell death, ${ }^{47,48,67}$ and therefore further work is required to confirm any direct role of GAPDH in the intrinsic mitochondrial pathway of apoptosis.

Evidence links GAPDH to several physiopathological models, particularly neurodegenerative disorders. ${ }^{68}$ Nuclear GAPDH has been found in fibroblasts and in vulnerable neurons of postmortem samples from patients affected with polyglutamine-repeat disorders such as Huntington's disease (HD) or dentatorubral-pallidoluysian atrophy, ${ }^{69,70}$ as well as from patients with Parkinson's disease ${ }^{71}$ and Alzheimer's disease (AD). ${ }^{72,73}$ Moreover, studies suggest that binding of the enzyme either to $\beta$-amyloid $(\mathrm{A} \beta)$ peptides or to mutant huntingtin, both directly involved in the development of these age-related disorders (AD and HD, respectively), regulates its cytotoxicity. ${ }^{74-76}$ In cell culture models, mutant huntingtin, with polyglutamine tracts in the $\mathrm{N}$-terminal region, elicits its cytotoxicity through the nuclear migration of its $\mathrm{N}$-terminal fragments. Overexpression of GAPDH or Siah1 enhances huntingtin nuclear translocation and cytotoxicity, whereas GAPDH mutants that cannot bind Siah1 prevented the translocation. ${ }^{74}$ Analyses of brain extracts from transgenic $A D$ mice coexpressing the mutated forms of human amyloid precursor protein and presenilin-1 gene, or postmortem samples from $A D$ patients, showed the accumulation of insoluble disulfide-linked multimers of GAPDH within neurons. $^{76}$ Furthermore, exposure of neuronal cells to $\mathrm{A} \beta$ promoted the formation of these insoluble aggregates of GAPDH to a similar extent as that induced by oxidative stress. ${ }^{56,76}$ Although $A \beta$-mediated neurotoxicity occurs through multiple biological pathways, the effect of $\mathrm{A} \beta$ on disulfide binding and the subsequent aggregation of an abundantly expressed protein such as GAPDH are likely to influence a number of these processes. Studies in cell lines and animal models showed that the protective effect of antidementia drugs, such as the cholinesterase inhibitors tacrine or donezepil, and deprenyl, a selective monoamine oxidase B inhibitor widely used in the treatment of Parkinson's disease, largely relied on their ability to interact with the GAPDH apoptotic cascade. ${ }^{73,77}$ These results prompted a clinical trial using the deprenyl derivative $\mathrm{TCH} 346$ in patients with amyotrophic lateral sclerosis; ${ }^{78}$ however, the trial showed no evidence of a beneficial effect on disease progression.

\section{GAPDH as a Prosurvival Factor}

Given its role as a proapoptotic agent, it is perhaps paradoxical that GAPDH is overexpressed in most human cancers. Dramatically elevated levels of glycolytic enzymes, including GAPDH expression, were observed in most human cancer types tested, ${ }^{79}$ and were often associated with reduced survival. ${ }^{80-83}$ Since Warburg's work in 1929, it has been known that cancer cells frequently upregulate glucose metabolism, resulting in a high uptake and use of glucose but moderate rates of mitochondrial respiration under aerobic conditions. Upregulation of glycolytic enzymes will lead to an enhancement of cell metabolism that has been shown to 


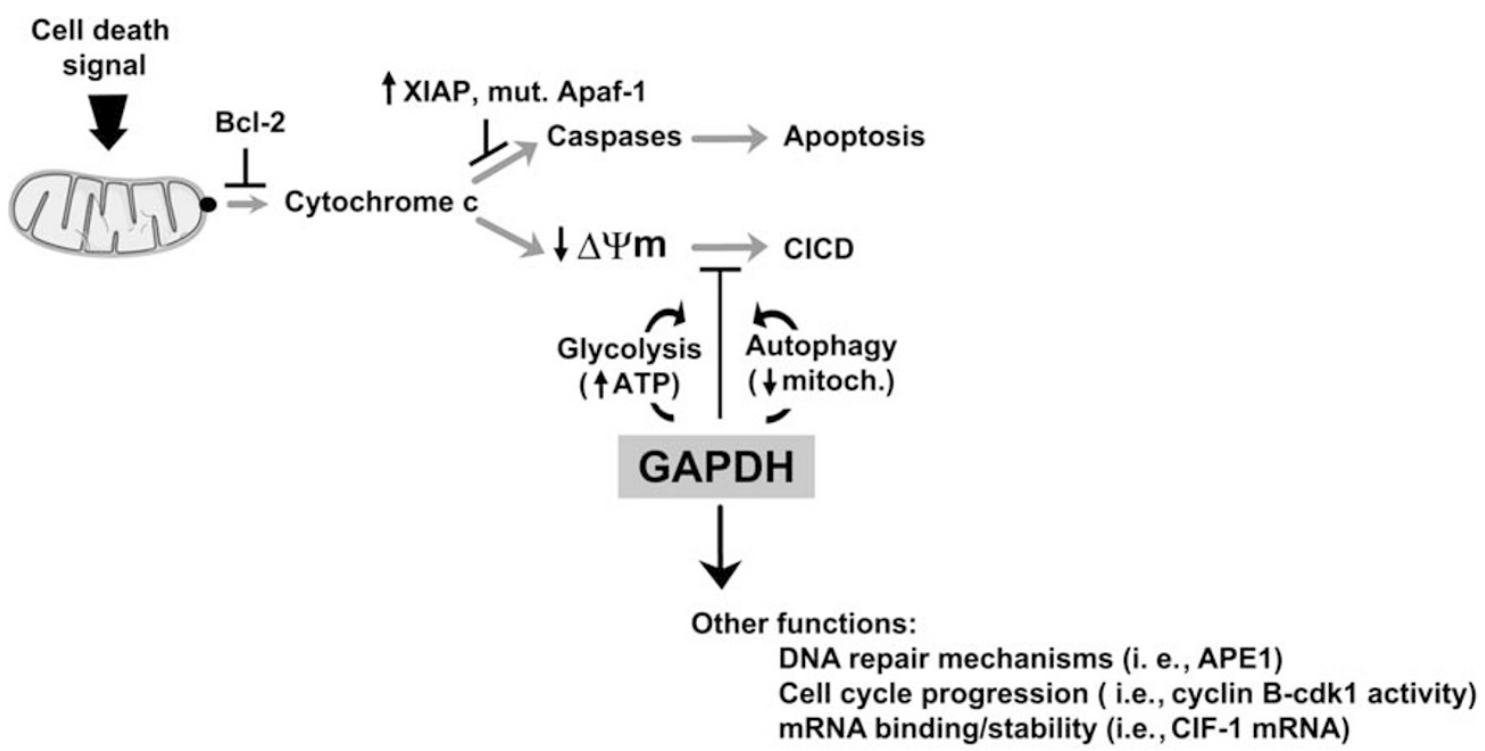

Figure 3 GAPDH as a prosurvival factor. Evasion of apoptosis is one of the hallmarks of human cancers, which promote tumor formation and progression, as well as treatment resistance. Signaling to cell death can be blocked by an increase in antiapoptotic molecules and/or by a decrease or defective function of proapoptotic proteins. The mitochondrial pathway may be impaired by overexpression of anti-apoptotic Bcl-2 proteins, as well as by Bax mutations or epigenetic silencing of Apaf-1. In addition, activation of downstream caspases can be blocked by high levels of IAPs (inhibitor of apoptosis proteins). However, besides caspase-dependent apoptosis, additional regulatory mechanisms of non-apoptotic modes of cell death must also be considered. When caspase activity is blocked, the decrease in mitochondrial membrane potential ( $\Delta \psi \mathrm{m})$ and function can result in caspase-independent cell death (CICD). Under these conditions, the increase in GAPDH levels inhibits cell death by simultaneously increasing ATP levels through glycolysis and stimulating autophagy-mediated clearance of permeabilized mitochondria. The involvement of GAPDH in other cellular processes may also contribute to its prosurvival role, including participation in DNA repair mechanisms, cell-cycle progression and mRNA binding and stability

correlate with increased tumor aggressiveness and poor patient prognosis in several cancers; ${ }^{84,85}$ however, the reason for this switch in the cell energetic status is debated. ${ }^{86}$

In a recent study by Colell et al. ${ }^{87}$ a novel role of elevated GAPDH was suggested. In this study, the authors used an unbiased genomic screen to identify proteins that protected cells from caspase-independent cell death $(\mathrm{CICD})^{88}$ (Figure 3). Apoptosis has a key role in suppressing oncogenesis, but under some conditions in which apoptosis is inhibited, a 'back-up death mechanism' can manifest. ${ }^{89}$ This alternative form of cell death was defined as CICD. ${ }^{88}$ Both apoptosis and $\mathrm{CICD}$ are triggered by mitochondrial outer membrane permeabilization (MOMP). ${ }^{89}$ Therefore, cells that resist cell death either do not undergo MOMP or have mechanisms to block or avoid both forms of cell death downstream of MOMP. Tumors frequently show such 'downstream defects', including mutation of caspases, lack of the adapter Apaf-1 or overexpression of the endogenous caspase inhibitor XIAP. ${ }^{90-92}$

Using a retroviral screen, GAPDH was identified as being able to protect cells from cell death after MOMP only when caspase activation was blocked or disrupted. ${ }^{87}$ GAPDH mutants that discriminate between glycolytic and non-glycolytic functions of the enzyme did not protect, but were effective when expressed together, indicating that two separate functions may be involved. One of these involves a novel role for GAPDH in autophagy induction. On CICD induction, GAPDH translocated to the nucleus, where it participated in an upregulation of the autophagy protein ATG $12{ }^{87}$ In support of this, GAPDH upregulation induced by bacterial $\mathrm{CpG}$ motifs in colon carcinoma cells was correlated with an increase in autophagy in vitro and in vivo. ${ }^{93}$ The elevated ATP levels as a result of glycolysis, and the function of autophagy to remove damaged mitochondria, both coordinated by GAPDH, cooperate in this protection toward CICD (Figure 3 ). However, in this study, protection from CICD involved GAPDH overexpression; therefore, further studies are required to unravel the mechanism whereby GAPDH protects cells from CICD under physiological conditions.

Very recently, under low-glucose conditions, GAPDH was found to inhibit mTORC1 signaling in an AMPK-independent manner by binding to Rheb. ${ }^{94}$ This is of particular interest in the context of GAPDH protection from CICD, as mTORC1 inhibition causes autophagy induction (among other functions). Therefore, investigating the role of $\mathrm{mTORC} 1$ signaling in protection from CICD is of interest.

The role of GAPDH as an anti-death molecule was further investigated in a model of chronic myeloid leukemia (CML) showing resistance to imatinib mesylate treatment. ${ }^{81}$ Imatinib mesylate is widely used for the treatment of patients with $\mathrm{CML}$, acting to induce apoptosis by counteracting Bcr-Abl activity. In all, 20-25\% patients develop resistance to imatinib for several reasons, but so far, the best-characterized mechanism is mutations in Bcr-Abl. Recently, imatinib was found to induce both apoptosis and CICD, and some imatinibresistant cells showed a spontaneous overexpression of GAPDH. Moderate knockdown of GAPDH did not affect the cellular metabolism, but sensitized these resistant cells to imatinib. Therefore, targeting GAPDH-mediated protection from CICD may be an innovative way of sensitizing 
imatinib-resistant CML patients. ${ }^{81}$ In this regard, a recent study suggested that a modulation of GAPDH levels affects prednisolone resistance in acute lymphoblastic leukemia cells. ${ }^{95}$

Roles for GAPDH in tumorigenesis and tumor progression are further supported by other studies. In ovarian cancer metastases, a strong coexpression of CSF-1 and its receptor was associated with poor prognosis, ${ }^{96}$ and GAPDH has recently been shown to bind and stabilize CSF-1 mRNA in this cell type. ${ }^{13}$ The authors speculated that one function of GAPDH in ovarian cancer is to increase the levels of CSF-1, an important cytokine in tumor progression.

Another protective role for GAPDH involves DNA repair and response to cytotoxic drugs. GAPDH can physically interact with APE1, an enzyme involved in the repair of spontaneous or drug-induced abasic sites in damaged DNA. ${ }^{97}$ This interaction results in a reactivation of APE1 endonuclease activity, thus preventing genomic instability resulting from aberrant structural changes caused by oxidative stress. Therefore, GAPDH can function to safeguard the genome by preventing APE1 inactivation on oxidative stress. Finally, GAPDH was found to be involved in cell-cycle regulation by modulating cyclin B-cdk1 activity, resulting in increased mitoses and accelerated cell-cycle progression. ${ }^{98}$

Most cancer cells exhibit increased glycolysis and use this metabolic pathway for ATP production. Therefore, inhibiting glycolysis could be an efficient way of targeting tumor cells while sparing normal tissue, and indeed several clinical trials have shown promising results using glycolytic inhibitors such as 2-deoxyglucose in combination with other anticancer agents (reviewed in Pelicano et al. ${ }^{99}$ and Scatena et al. ${ }^{100}$ ). Recent studies have shown that koningic acid, a selective inhibitor of GAPDH, kills a broad range of highly glycolytic cell lines through growth inhibition and CICD. ${ }^{101}$ Thus, understanding the biological functions of GAPDH beyond glycolysis will likely improve our ability to effectively target this enzyme in cancer therapy.

Concluding Remarks. There is mounting evidence that cell death is initiated after the synthesis of a new GAPDH protein and its subsequent nuclear appearance. However, the same events have also been observed during cell proliferation and/ or transformation. Indeed, most cells exhibit some GAPDH translocation to the nucleus on cell cycle, and some, such as lymphocytes, naturally upregulate it on activation, without dying. How can this paradox be reconciled? It may well be that transformation requires that cells bypass a GAPDHmediated metabolic checkpoint. One possible model would be that cells that normally derive energy from oxidation have a GAPDH-mediated checkpoint that can kill them if, because of sudden changes in signaling (indicative of transforming events), they engage elevated glycolysis. However, mechanisms must exist to overcome or bypass this in cells that make such a shift as a part of normal physiology. One of these might be linked to ROS production. GAPDH is a redoxsensitive protein that is inactivated by ROS. Therefore, in conditions that induce a mild ROS production (lymphocyte activation), GAPDH upregulation may be protective for the cell. However, in the presence of higher amounts of ROS, such as those observed under conditions of mitochondrial dysfunction, oxidation of $\mathrm{Cys}^{152}$ may lead to a GAPDH inhibition, participating in cell death. As we know very little regarding the manner in which GAPDH engages apoptosis or prevents CICD, the actual mechanisms involved in the above scenario remain obscure.

Acknowledgements. We thank Dr. SW Tait (St. Jude Children's Research Institute, Memphis, TN, USA) for critical reading of this paper. This work was supported in part by l'Association pour la Recherche sur le Cancer, by l'Agence Nationnal de la Recherche, la Fondation de France, Plan Nacional I + D SAF200804974 and by grants from The U.S. National Institutes of Health. J-E.R. is a recipient of a contrat d'interface INSERM-CHU de Nice.

1. Sirover MA. New insights into an old protein: the functional diversity of mammalian glyceraldehyde-3-phosphate dehydrogenase. Biochim Biophys Acta 1999; 1432: 159-184.

2. Sirover MA. New nuclear functions of the glycolytic protein, glyceraldehyde-3-phosphate dehydrogenase, in mammalian cells. J Cell Biochem 2005; 95: 45-52.

3. Morero RD, Vinals AL, Bloj B, Farias RN. Fusion of phospholipid vesicles induced by muscle glyceraldehyde-3-phosphate dehydrogenase in the absence of calcium. Biochemistry 1985; 24: 1904-1909.

4. Robbins AR, Ward RD, Oliver C. A mutation in glyceraldehyde 3-phosphate dehydrogenase alters endocytosis in CHO cells. J Cell Biol 1995; 130: 1093-1104.

5. Nakagawa T, Hirano Y, Inomata A, Yokota S, Miyachi K, Kaneda M et al. Participation of a fusogenic protein, glyceraldehyde-3-phosphate dehydrogenase, in nuclear membrane assembly. J Biol Chem 2003; 278: 20395-20404

6. Tisdale EJ, Kelly $C$, Artalejo CR. Glyceraldehyde-3-phosphate dehydrogenase interacts with Rab2 and plays an essential role in endoplasmic reticulum to Golgi transport exclusive of its glycolytic activity. J Biol Chem 2004; 279: 54046-54052.

7. Walsh JL, Keith TJ, Knull HR. Glycolytic enzyme interactions with tubulin and microtubules. Biochim Biophys Acta 1989; 999: 64-70.

8. Huitorel P, Pantaloni D. Bundling of microtubules by glyceraldehyde-3-phosphate dehydrogenase and its modulation by ATP. Eur J Biochem 1985; 150: 265-269.

9. Tisdale EJ, Azizi F, Artalejo CR. Rab2 utilizes glyceraldehyde-3-phosphate dehydrogenase and protein kinase $\mathrm{C} \backslash\{$ iotal $\}$ to associat. J Biol Chem 2009; 284 : $5876-5884$.

10. Singh R, Green MR. Sequence-specific binding of transfer RNA by glyceraldehyde-3phosphate dehydrogenase. Science 1993; 259: 365-368.

11. Nagy E, Henics T, Eckert M, Miseta A, Lightowlers RN, Kellermayer M. Identification of the $N A D(+)$-binding fold of glyceraldehyde-3-phosphate dehydrogenase as a novel RNAbinding domain. Biochem Biophys Res Commun 2000; 275: 253-260.

12. Rodriguez-Pascual F, Redondo-Horcajo M, Magan-Marchal N, Lagares D, Martinez-Ruiz A, Kleinert $\mathrm{H}$ et al. Glyceraldehyde-3-phosphate dehydrogenase regulates endothelin-1 expression by a novel, redox-sensitive mechanism involving mRNA stability. Mol Cell Biol 2008; 28: 7139-7155.

13. Zhou $Y$, Yi X, Stoffer JB, Bonafe N, Gilmore-Hebert M, McAlpine $J$ et al. The multifunctional protein glyceraldehyde-3-phosphate dehydrogenase is both regulated and controls colony-stimulating factor-1 messenger RNA stability in ovarian cancer. $\mathrm{Mol}$ Cancer Res 2008; 6: 1375-1384

14. Zheng $L$, Roeder RG, Luo Y. S phase activation of the histone H2B promoter by OCA-S, a coactivator complex that contains GAPDH as a key component. Cell 2003; 114: 255-266.

15. Mitsuzawa H, Kimura M, Kanda E, Ishihama A. Glyceraldehyde-3-phosphate dehydrogenase and actin associate with RNA polymerase II and interact with its Rpb7 subunit. FEBS Lett 2005; 579: 48-52.

16. Meyer-Siegler K, Mauro DJ, Seal G, Wurzer J, deRiel JK, Sirover MA. A human nuclear uracil DNA glycosylase is the $37-\mathrm{kDa}$ subunit of glyceraldehyde-3-phosphate dehydrogenase. Proc Natl Acad Sci USA 1991; 88: 8460-8464.

17. Krynetski EY, Krynetskaia NF, Gallo AE, Murti KG, Evans WE. A novel protein complex distinct from mismatch repair binds thioguanylated DNA. Mol Pharmacol 2001; 59: 367-374.

18. Sundararaj KP, Wood RE, Ponnusamy S, Salas AM, Szulc Z, Bielawska A et al. Rapid shortening of telomere length in response to ceramide involves the inhibition of telomere binding activity of nuclear glyceraldehyde-3-phosphate dehydrogenase. J Biol Chem 2004; 279: 6152-6162.

19. Meyer-Siegler K, Rahman-Mansur N, Wurzer JC, Sirover MA. Proliferative dependent regulation of the glyceraldehyde-3-phosphate dehydrogenase/uracil DNA glycosylase gene in human cells. Carcinogenesis 1992; 13: 2127-2132.

20. Mansur NR, Meyer-Siegler K, Wurzer JC, Sirover MA. Cell cycle regulation of the glyceraldehyde-3-phosphate dehydrogenase/uracil DNA glycosylase gene in normal human cells. Nucleic Acids Res 1993; 21: 993-998.

21. Netzker R, Hermfisse U, Wein $\mathrm{KH}$, Brand $\mathrm{K}$. Expression of glycolytic isozymes in rat thymocytes during cell cycle progression. Biochim Biophys Acta 1994; 1224: 371-376. 
22. Graven KK, Yu Q, Pan D, Roncarati JS, Farber HW. Identification of an oxygen responsive enhancer element in the glyceraldehyde-3-phosphate dehydrogenase gene. Biochim Biophys Acta 1999; 1447: 208-218.

23. Alexander-Bridges M, Dugast I, Ercolani L, Kong XF, Giere L, Nasrin N. Multiple insulinresponsive elements regulate transcription of the GAPDH gene. Adv Enzyme Regul 1992; 32: 149-159.

24. Folkman J, Hahnfeldt P, Hlatky L. Cancer: looking outside the genome. Nat Rev Mol Cell Biol 2000; 1: 76-79.

25. Lartigau E, Le Ridant AM, Lambin P, Weeger P, Martin L, Sigal R et al. Oxygenation of head and neck tumors. Cancer 1993; 71: 2319-2325.

26. Lartigau E, Randrianarivelo $\mathrm{H}$, Avril MF, Margulis A, Spatz A, Eschwege $\mathrm{F}$ et al. Intratumoral oxygen tension in metastatic melanoma. Melanoma Res 1997; 7: 400-406.

27. Semenza GL. HIF-1: mediator of physiological and pathophysiological responses to hypoxia. J Appl Physiol 2000; 88: 1474-1480.

28. Firth JD, Ebert BL, Pugh CW, Ratcliffe PJ. Oxygen-regulated control elements in the phosphoglycerate kinase 1 and lactate dehydrogenase A genes: similarities with the erythropoietin 3' enhancer. Proc Natl Acad Sci USA 1994; 91: 6496-6500.

29. Semenza GL, Roth PH, Fang HM, Wang GL. Transcriptional regulation of genes encoding glycolytic enzymes by hypoxia-inducible factor 1. J Biol Chem 1994; 269 : 23757-23763.

30. Graven KK, Troxler RF, Kornfeld H, Panchenko MV, Farber HW. Regulation of endothelial cell glyceraldehyde-3-phosphate dehydrogenase expression by hypoxia. J Biol Chem 1994; 269: 24446-24453.

31. Graven KK, Bellur D, Klahn BD, Lowrey SL, Amberger E. HIF-2alpha regulates glyceraldehyde-3-phosphate dehydrogenase expression in endothelial cells. Biochim Biophys Acta 2003; 1626: 10-18.

32. Cuervo AM, Terlecky SR, Dice JF, Knecht E. Selective binding and uptake of ribonuclease $A$ and glyceraldehyde-3-phosphate dehydrogenase by isolated rat liver lysosomes. J Biol Chem 1994; 269: 26374-26380.

33. Cuervo AM. Autophagy: in sickness and in health. Trends Cell Biol 2004; 14: 70-77.

34. Bandyopadhyay U, Kaushik S, Varticovski L, Cuervo AM. The chaperone-mediated autophagy receptor organizes in dynamic protein complexes at the lysosomal membrane. Mol Cell Biol 2008; 28: 5747-5763.

35. Singh P, Salih M, Leddy JJ, Tuana BS. The muscle-specific calmodulin-dependent protein kinase assembles with the glycolytic enzyme complex at the sarcoplasmic reticulum and modulates the activity of glyceraldehyde-3-phosphate dehydrogenase in a Ca2+/calmodulin-dependent manner. J Biol Chem 2004; 279: 35176-35182.

36. Tisdale EJ. Glyceraldehyde-3-phosphate dehydrogenase is phosphorylated by protein kinase Ciota /lambda and plays a role in microtubule dynamics in the early secretory pathway. J Biol Chem 2002; 277: 3334-3341.

37. Tisdale EJ, Artalejo CR. Src-dependent aprotein kinase C iota/lambda (aPKCiota/ lambda) tyrosine phosphorylation is required for aPKCiota/lambda association with Rab2 and glyceraldehyde-3-phosphate dehydrogenase on pre-golgi intermediates. J Biol Chem 2006; 281: 8436-8442

38. Hara MR, Agrawal N, Kim SF, Cascio MB, Fujimuro M, Ozeki Y et al. S-nitrosylated GAPDH initiates apoptotic cell death by nuclear translocation following Siah1 binding. Nat Cell Biol 2005; 7: 665-674.

39. Mohr S, Stamler JS, Brune B. Posttranslational modification of glyceraldehyde-3phosphate dehydrogenase by S-nitrosylation and subsequent NADH attachment. J Biol Chem 1996; 271: 4209-4214

40. Batthyany C, Schopfer FJ, Baker PR, Duran R, Baker LM, Huang Y et al. Reversible posttranslational modification of proteins by nitrated fatty acids in vivo. J Biol Chem 2006; 281 20450-20463.

41. Mohr S, Hallak H, de Boitte A, Lapetina EG, Brune B. Nitric oxide-induced S-glutathionylation and inactivation of glyceraldehyde-3-phosphate dehydrogenase. J Biol Chem 1999; 274: 9427-9430.

42. Little C, O'Brien PJ. Mechanism of peroxide-inactivation of the sulphydryl enzyme glyceraldehyde-3-phosphate dehydrogenase. Eur J Biochem 1969; 10: 533-538.

43. Souza JM, Radi R. Glyceraldehyde-3-phosphate dehydrogenase inactivation by peroxynitrite. Arch Biochem Biophys 1998; 360: 187-194.

44. Dastoor Z, Dreyer JL. Potential role of nuclear translocation of glyceraldehyde-3phosphate dehydrogenase in apoptosis and oxidative stress. J Cell Sci 2001; 114 (Pt 9): 1643-1653.

45. Ishitani R, Kimura M, Sunaga K, Katsube N, Tanaka M, Chuang DM. An antisense oligodeoxynucleotide to glyceraldehyde-3-phosphate dehydrogenase blocks ageinduced apoptosis of mature cerebrocortical neurons in culture. J Pharmacol Exp Ther 1996; 278: 447-454.

46. Ishitani $R$, Sunaga $K$, Hirano $A$, Saunders $P$, Katsube $N$, Chuang DM. Evidence that glyceraldehyde-3-phosphate dehydrogenase is involved in age-induced apoptosis in mature cerebellar neurons in culture. J Neurochem 1996; 66: 928-935.

47. Ishitani R, Tanaka M, Sunaga K, Katsube N, Chuang DM. Nuclear localization of overexpressed glyceraldehyde-3-phosphate dehydrogenase in cultured cerebellar neurons undergoing apoptosis. Mol Pharmacol 1998; 53: 701-707.

48. Saunders PA, Chalecka-Franaszek E, Chuang DM. Subcellular distribution of glyceraldehyde-3-phosphate dehydrogenase in cerebellar granule cells undergoing cytosine arabinoside-induced apoptosis. J Neurochem 1997; 69: 1820-1828.
49. Sawa A, Khan AA, Hester LD, Snyder SH. Glyceraldehyde-3-phosphate dehydrogenase: nuclear translocation participates in neuronal and nonneuronal cell death. Proc Natl Acad Sci USA 1997; 94: 11669-11674.

50. Schmitz HD. Reversible nuclear translocation of glyceraldehyde-3-phosphate dehydrogenase upon serum depletion. Eur J Cell Biol 2001; 80: 419-427.

51. Brown VM, Krynetski EY, Krynetskaia NF, Grieger D, Mukatira ST, Murti KG et al. A novel CRM1-mediated nuclear export signal governs nuclear accumulation of glyceraldehyde-3-phosphate dehydrogenase following genotoxic stress. J Biol Chem 2004; 279: 5984-5992.

52. Park J, Han D, Kim K, Kang Y, Kim Y. O-GlcNAcylation disrupts glyceraldehyde-3phosphate dehydrogenase homo-tetramer formation and mediates its nuclear translocation. Biochim Biophys Acta 2009; 1794: 254-262.

53. Ishitani R, Chuang DM. Glyceraldehyde-3-phosphate dehydrogenase antisense oligodeoxynucleotides protect against cytosine arabinonucleoside-induced apoptosis in cultured cerebellar neurons. Proc Natl Acad Sci USA 1996; 93: 9937-9941.

54. Maruyama W, Akao Y, Youdim MB, Davis BA, Naoi M. Transfection-enforced Bcl-2 overexpression and an anti-Parkinson drug, rasagiline, prevent nuclear accumulation of glyceraldehyde-3-phosphate dehydrogenase induced by an endogenous dopaminergic neurotoxin, N-methyl(R)salsolinol. J Neurochem 2001; 78: 727-735.

55. Saunders PA, Chen RW, Chuang DM. Nuclear translocation of glyceraldehyde-3phosphate dehydrogenase isoforms during neuronal apoptosis. J Neurochem 1999; 72 : 925-932.

56. Nakajima H, Amano W, Fujita A, Fukuhara A, Azuma YT, Hata F et al. The active site cysteine of the proapoptotic protein glyceraldehyde-3-phosphate dehydrogenase is essential in oxidative stress-induced aggregation and cell death. J Biol Chem 2007; 282 26562-26574

57. Coan C, Ji JY, Hideg K, Mehlhorn RJ. Protein sulfhydryls are protected from irreversible oxidation by conversion to mixed disulfides. Arch Biochem Biophys 1992; 295 369-378.

58. Cotgreave IA, Gerdes R, Schuppe-Koistinen I, Lind C. S-glutathionylation of glyceraldehyde-3-phosphate dehydrogenase: role of thiol oxidation and catalysis by glutaredoxin. Methods Enzymol 2002; 348: 175-182

59. Ralser M, Wamelink MM, Kowald A, Gerisch B, Heeren G, Struys EA et at Dynamic rerouting of the carbohydrate flux is key to counteracting oxidative stress. J Biol 2007; 6: 10.

60. Morigasaki S, Shimada K, Ikner A, Yanagida M, Shiozaki K. Glycolytic enzyme GAPDH promotes peroxide stress signaling through multistep phosphorelay to a MAPK cascade. Mol Cell 2008; 30: 108-113.

61. Hara MR, Cascio MB, Sawa A. GAPDH as a sensor of NO stress. Biochim Biophys Acta 2006; 1762: 502-509.

62. Hara MR, Snyder SH. Nitric oxide-GAPDH-Siah: a novel cell death cascade. Cell Mol Neurobiol 2006; 26: 527-538.

63. Sen N, Hara MR, Kornberg MD, Cascio MB, Bae BI, Shahani $\mathrm{N}$ et al. Nitric oxide-induced nuclear GAPDH activates p300/CBP and mediates apoptosis. Nat Cell Biol 2008; 10 . 866-873.

64. Fiucci G, Beaucourt S, Duflaut D, Lespagnol A, Stumptner-Cuvelette P, Geant A et al. Siah-1b is a direct transcriptional target of $\mathrm{p53}$ : identification of the functional p53 responsive element in the siah-1b promoter. Proc Natl Acad Sci USA 2004; 101 3510-3515.

65. Chen RW, Saunders PA, Wei H, Li Z, Seth P, Chuang DM. Involvement of glyceraldehyde-3-phosphate dehydrogenase (GAPDH) and p53 in neuronal apoptosis: evidence that GAPDH is upregulated by p53. J Neurosci 1999; 19: 9654-9662.

66. Du ZX, Wang HQ, Zhang HY, Gao DX. Involvement of glyceraldehyde-3-phosphate dehydrogenase in tumor necrosis factor-related apoptosis-inducing ligand-mediated death of thyroid cancer cells. Endocrinology 2007; 148: 4352-4361.

67. Tarze A, Deniaud A, Le Bras M, Maillier E, Molle D, Larochette N et al. GAPDH, a novel regulator of the pro-apoptotic mitochondrial membrane permeabilization. Oncogene 2007; 26: 2606-2620.

68. Chuang DM, Hough C, Senatorov VV. Glyceraldehyde-3-phosphate dehydrogenase, apoptosis, and neurodegenerative diseases. Annu Rev Pharmacol Toxicol 2005; 45: 269-290.

69. Mazzola JL, Sirover MA. Alteration of nuclear glyceraldehyde-3-phosphate dehydrogenase structure in Huntington's disease fibroblasts. Brain Res Mol Brain Res 2002; 100: 95-101.

70. Shiozawa M, Fukutani Y, Arai N, Cairns NJ, Mizutani T, Isaki K et al. Glyceraldehyde 3phosphate dehydrogenase and endothelin-1 immunoreactivity is associated with cerebral white matter damage in dentatorubral-pallidoluysian atrophy. Neuropathology 2003; 23 36-43.

71. Tatton NA. Increased caspase 3 and Bax immunoreactivity accompany nuclear GAPDH translocation and neuronal apoptosis in Parkinson's disease. Exp Neurol 2000 166: $29-43$

72. Mazzola JL, Sirover MA. Subcellular alteration of glyceraldehyde-3-phosphate dehydrogenase in Alzheimer's disease fibroblasts. J Neurosci Res 2003; 71: 279-285.

73. Tsuchiya K, Tajima H, Yamada M, Takahashi H, Kuwae T, Sunaga K et al. Disclosure of a pro-apoptotic glyceraldehyde-3-phosphate dehydrogenase promoter: anti-dementia drugs depress its activation in apoptosis. Life Sci 2004; 74: 3245-3258. 
74. Bae BI, Hara MR, Cascio MB, Wellington CL, Hayden MR, Ross CA et al. Mutant huntingtin: nuclear translocation and cytotoxicity mediated by GAPDH. Proc Natl Acad Sc USA 2006; 103: 3405-3409.

75. Verdier Y, Foldi I, Sergeant N, Fulop L, Penke Z, Janaky T et al. Characterization of the interaction between Abeta 1-42 and glyceraldehyde phosphodehydrogenase. J Pept Sc 2008; 14: 755-762.

76. Cumming RC, Schubert D. Amyloid-beta induces disulfide bonding and aggregation of GAPDH in Alzheimer's disease. Faseb J 2005; 19: 2060-2062.

77. Hara MR, Thomas B, Cascio MB, Bae BI, Hester LD, Dawson VL et al. Neuroprotection by pharmacologic blockade of the GAPDH death cascade. Proc Natl Acad Sci USA 2006; 103: 3887-3889.

78. Miller R, Bradley W, Cudkowicz M, Hubble J, Meininger V, Mitsumoto $\mathrm{H}$ et al. Phase II/II randomized trial of TCH346 in patients with ALS. Neurology 2007; 69: 776-784.

79. Altenberg B, Greulich KO. Genes of glycolysis are ubiquitously overexpressed in 24 cancer classes. Genomics 2004; 84: 1014-1020.

80. Epner DE, Coffey DS. There are multiple forms of glyceraldehyde-3-phosphate dehydrogenase in prostate cancer cells and normal prostate tissue. Prostate 1996; 28 372-378.

81. Lavallard VJ, Pradelli LA, Paul A, Beneteau M, Jacquel A, Auberger P et al. Modulation of caspase-independent cell death leads to resensitization of imatinib mesylate-resistan cells. Cancer Res 2009; 69: 3013-3020.

82. Lu H, Zhang Y, Roberts DD, Osborne CK, Templeton NS. Enhanced gene expression in breast cancer cells in vitro and tumors in vivo. Mol Ther 2002; 6: 783-792.

83. Revillion F, Pawlowski V, Hornez L, Peyrat JP. Glyceraldehyde-3-phosphate dehydrogenase gene expression in human breast cancer. Eur $J$ Cancer 2000; 36 1038-1042.

84. Detterbeck FC, Vansteenkiste JF, Morris DE, Dooms CA, Khandani AH, Socinski MA Seeking a home for a PET, part 3: emerging applications of positron emission tomography imaging in the management of patients with lung cancer. Chest 2004; 126 : 1656-1666.

85. Strauss LG, Conti PS. The applications of PET in clinical oncology. J Nucl Med 1991; 32 623-648; discussion 649-650.

86. Vander Heiden MG, Cantley LC, Thompson CB. Understanding the Warburg effect: the metabolic requirements of cell proliferation. Science 2009; 324: 1029-1033.

87. Colell A, Ricci JE, Tait S, Milasta S, Maurer U, Bouchier-Hayes L et al. GAPDH and autophagy preserve survival after apoptotic cytochrome $c$ release in the absence of caspase activation. Cell 2007; 129: 983-997.

88. Chipuk JE, Green DR. Do inducers of apoptosis trigger caspase-independent cell death? Nat Rev Mol Cell Biol 2005; 6: 268-275.

89. Tait SW, Green DR. Caspase-independent cell death: leaving the set without the final cut Oncogene 2008; 27: 6452-6461.

90. Liu JR, Opipari AW, Tan L, Jiang Y, Zhang Y, Tang $\mathrm{H}$ et al. Dysfunctional apoptosome activation in ovarian cancer: implications for chemoresistance. Cancer Res 2002; 62 924-931.
91. Schimmer AD, Dalili S, Batey RA, Riedl SJ. Targeting XIAP for the treatment of malignancy. Cell Death Differ 2006; 13: 179-188.

92. Soengas MS, Capodieci P, Polsky D, Mora J, Esteller M, Opitz-Araya X et al. Inactivation of the apoptosis effector Apaf-1 in malignant melanoma. Nature 2001; 409: 207-211.

93. Bertin S, Samson M, Pons C, Guigonis JM, Gavelli A, Baque P et al. Comparative proteomics study reveals that bacterial $\mathrm{CpG}$ motifs induce tumor cell autophagy in vitro and in vivo. Mol Cell Proteomics 2008; 12: 2311-2322.

94. Lee MN, Ha SH, Kim J, Koh A, Lee CS, Kim JH et al. Glycolytic flux signals to mTOR through glyceraldehyde-3-phosphate dehydrogenase-mediated regulation of Rheb. Mol Cell Biol 2009; 29: 3991-4001.

95. Hulleman E, Kazemier KM, Holleman A, VanderWeele DJ, Rudin CM, Broekhuis MJ et al. Inhibition of glycolysis modulates prednisolone resistance in acute lymphoblastic leukemia cells. Blood 2009; 113: 2014-2021.

96. Chambers SK, Kacinski BM, Ivins CM, Carcangiu ML. Overexpression of epithelial macrophage colony-stimulating factor (CSF-1) and CSF-1 receptor: a poor prognostic factor in epithelial ovarian cancer, contrasted with a protective effect of stromal CSF-1. Clin Cancer Res 1997; 3: 999-1007.

97. Azam S, Jouvet $\mathrm{N}$, Jilani A, Vongsamphanh $\mathrm{R}$, Yang $\mathrm{X}$, Yang $\mathrm{S}$ et al. Human glyceraldehyde-3-phosphate dehydrogenase plays a direct role in reactivating oxidized forms of the DNA repair enzyme APE1. J Biol Chem 2008; 283: 30632-30641.

98. Carujo S, Estanyol JM, Ejarque A, Agell N, Bachs O, Pujol MJ. Glyceraldehyde 3phosphate dehydrogenase is a SET-binding protein and regulates cyclin B-cdk1 activity. Oncogene 2006; 25: 4033-4042.

99. Pelicano H, Martin DS, Xu RH, Huang P. Glycolysis inhibition for anticancer treatment. Oncogene 2006; 25: 4633-4646.

100. Scatena R, Bottoni P, Pontoglio A, Mastrototaro L, Giardina B. Glycolytic enzyme inhibitors in cancer treatment. Expert Opin Investig Drugs 2008; 17: 1533-1545.

101. Kumagai S, Narasaki R, Hasumi K. Glucose-dependent active ATP depletion by koningic acid kills high-glycolytic cells. Biochem Biophys Res Commun 2008; 365: 362-368.

102. Ishitani R, Sunaga K, Tanaka M, Aishita H, Chuang DM. Overexpression of glyceraldehyde-3-phosphate dehydrogenase is involved in low $\mathrm{K}+$-induced apoptosis but not necrosis of cultured cerebellar granule cells. Mol Pharmacol 1997; 51: 542-550.

103. Fukuhara Y, Takeshima T, Kashiwaya Y, Shimoda K, Ishitani R, Nakashima K. GAPDH knockdown rescues mesencephalic dopaminergic neurons from MPP+ -induced apoptosis. Neuroreport 2001; 12: 2049-2052.

104. Maruyama W, Oya-Ito T, Shamoto-Nagai M, Osawa T, Naoi M. Glyceraldehyde-3phospate dehydrogenase is translocated into nuclei through Golgi apparatus during apoptosis induced by 6-hydroxydopamine in human dopaminergic SH-SY5Y cells. Neurosci Lett 2002; 321: 29-32.

105. Barbini L, Rodriguez J, Dominguez F, Vega F. Glyceraldehyde-3-phosphate dehydrogenase exerts different biologic activities in apoptotic and proliferating hepatocytes according to its subcellular localization. Mol Cell Biochem 2007; 300: 19-28. 